\title{
Nonalcoholic fatty liver disease (NAFLD), more than a liver disease
}

HEADINGS - Non-alcoholic fatty liver disease. Hepatocellular carcinoma. Type 2 diabetes mellitus. Cardiovascular diseases. Chronic renal insufficiency.

Nonalcoholic fatty liver disease (NAFLD) is considered the commonest cause of liver disease ${ }^{(1)}$, with highest prevalence in the Middle East and Latin America and lowest in Africa ${ }^{(2)}$. In the USA, Hispanics have the highest prevalence of NAFLD, then Caucasians and less in African Americans ${ }^{(3)}$. Among some especial patient populations the prevalence of NAFLD varies, in obese patients undergoing bariatric surgery has been estimated to be up to $90 \%$ and among diabetic patients the prevalence of NAFLD ranges from $40 \%$ to $71 \% 0^{(4,5)}$.

Nonalcoholic steatohepatitis (NASH), the progressive form of NAFLD, is growing in medical importance as one of the main etiologies for chronic liver diseases and hepatocellular carcinoma, what can be reflected by the prevalence of patients with the disease referred to liver transplantation. In 2009, NASH-related cirrhosis was the third most common indication for liver transplantation in the United States, behind hepatitis $\mathrm{C}$ and alcoholic liver disease, and was the only liver-related transplant indication that continued to increase ${ }^{(6)}$. More recent data, confirmed this rate of growing, with NASH surpassing hepatitis $\mathrm{C}$ as the main cause of liver transplant indication in the USA among patients under 50 years old ${ }^{(7)}$. Also considering indication of liver transplant for hepatocellular carcinoma, NASH is also the most rapidly increasing etiology among indications for liver transplant due to hepatocellular carcinoma ${ }^{(8)}$ and, with the decline in hepatic transplantation for hepatitis $\mathrm{C}^{(9)}$, it is expected NASH to become the leading cause of HCC-related liver transplantation in USA and in other parts of the world ${ }^{(10,11)}$. The Latin America multicenter cohort is a good example of the progression of NAFLD as one of the main causes of HCC submitted to liver transplantation. Comparing the periods including years 2005-2006 with years 2011-2012 there was a 6-fold increase in NAFLD-related HCC whereas for those cases associated with $\mathrm{HCV}$ there was a 2-fold decrease in the same period of time ${ }^{(10)}$.

Although these impressive numbers stressed the importance of NAFLD/NASH as a liver disease, its importance in medical care goes far beyond, thanks to its interaction with type 2 diabetes mellitus and cardiovascular diseases and other diseases.

Insulin resistance(IR) is present in virtually all patients with NAFLD $^{(12)}$ and glucose intolerance is frequently found along with overt diabetes mellitus type $2(\mathrm{~T} 2 \mathrm{DM})^{(5,12-14)}$. While insulin resistance assessed by Homeostatic Model for Insulin Resistance (HOMAIR) is associated with significant increase in the prevalence of steatohepatitis in NAFLD, T2DM is associated with more rapidly evolution of the disease to NASH and hepatic fibrosis ${ }^{(15,16)}$. In a prospective evaluation of 180 patients with T2DM, Leite et al. ${ }^{(16)}$ found NAFLD in $69 \%$ of them, with $54 \%$ of them with NASH and $28 \%$ already with liver fibrosis grade 2 or more according to the NASH Clinical Research Network scoring system. But if diabetes aggravates NAFLD leading to more severe forms of steatohepatitis, fibrosis and even HCC, NAFLD itself can modify the natural history of T2DM. Patients with NAFLD progress to T2DM significantly more frequent than the general population, and most non diabetic NAFLD patients will be diagnosed with diabetes or impaired glucose tolerance (IGT) in a long term follow-up of the disease ${ }^{(17)}$. In the follow-up of 5 years of Corean individuals, those with NAFLD diagnosed by ultrasound had a 2.78 times more chance to develop future diabetes than patients without NAFLD. For those who progressed from mild to moderate or advanced NAFLD the risk of developing T2DM was 7.38 times more, even when data were adjusted for confounding factors (as age, gender, BMI, glucose, dislipemia, physical activity). More interesting no increased risk for this outcome was observed for those individuals in whom NAFLD regressed along the years.

Manatovani et al. ${ }^{(18)}$ in a recent meta-analysis with 19 observational cohort studies confirmed that NAFLD was significantly associated with 2.2 times increased of the risk of incident diabetes [random-effects Hazard ratio (HR) 2.22, 95\% CI 1.84-2.60] and this random-effects HR was independent of a relatively large number of common risk factors and potential confounders.

Finally, in patients with T2DM, the presence of NAFLD is associated with an increased risk of overall death, and is a independent predictor of death together with presence of ischemic heart disease and duration of diabetes ${ }^{(19)}$.

Dyslipidemia is frequently found in $\operatorname{NAFLD}^{(2,5,7)}$ and is characterized by increased serum levels of triglycerides (TG) and lowdensity lipoprotein cholesterol (LDL-C) levels and by decreased high-density lipoprotein cholesterol (HDL-C) concentrations. As suggested by Ray G, Agarwala T in this number of Arq Gastroenterol, in nondiabetic abstinent patients, dyslipidemias could be a prime contributor to the development of NAFLD ${ }^{(20)}$. On the other hand, since dyslipidemia, T2DM and other features of metabolic syndrome are frequently found together in some NAFLD patient it is not surprising that cardiovascular disease become an important cause of death among these patients. In fact, studies on the natural history of patients with NAFLD, indicate the cardiovascular disease as the number one cause of death during the follow up of these patients, mainly among those with $\mathrm{NASH}^{(16,21,22)}$. Although 
NASH was the former histological feature to be related with survival, further studies showed that fibrosis (especially advanced or significant fibrosis) was more associated with increment in death from liver disease and also from non-hepatic causes than the necroinflammatory component ${ }^{(22-24)}$. Such observations, highlights the importance of studies on the natural history of the disease and its progression toward liver fibrosis, as observed in the article from Castro et al. in this issue of Arq Gastroenterol ${ }^{(25)}$.

As it was pointed out before for T2DM, there is a counterpart where NAFLD can interfere with cardiovascular disease. In fact atherosclerosis is more prevalent and progress more rapidly in patients with NAFLD, than in patients with the same risk factors but without the liver disease. In a meta-analysis with pooled data from 20 studies (8652 patients with NASH plus 10662 controls) there was a significant increase in intimal medial thickness (C-IMT) and in carotid plaque prevalence among patients with NAFLD ${ }^{(26)}$. In this study, even among children the C-IMT was more pronouncied in those with liver disease.

When patients with diabetes, obesity, metabolic syndrome or other risk factors for cardiovascular disease with or without NAFLD are compared, there is a clear increase in the incidence of non-fatal but not for fatal cardiovascular disease (CVD) among the first group of patients ${ }^{(27,28)}$. One of the possible explanations for that could be that in most studies the diagnostic of NAFLD was made only by abdominal ultrasound that is not able to identify liver fibrosis, and as NASH-associated fibrosis account for the minority of patients with NAFLD in the general population, a proper evaluation of risk related to the presence of fibrosis could not be identified. In contrast, when biopsy, noninvasive methods or indirect evidence of more aggressive forms of the disease are identified, a significant increase in the number of fatal CVD events could be found ${ }^{(28)}$.

Cardiac diastolic dysfunction, altered electric conduction/ arrhythmia and aortic valve calcification are others cardiological features that have been associated with NAFLD, but further research is needed to corroborate these findings ${ }^{(29)}$.

Since T2DM, CVD and NAFLD are all related to metabolic syndrome it seems, as proposed by Francque et al. ${ }^{(30)}$, that there is a complex interplay among these three diseases, where NAFLD can be considered the consequence of mechanism driven by the other components of the metabolic syndrome. However, reciprocal crosstalk exists, wherein the liver may actually drive to diabetes mellitus or cardiovascular disease. These synergetic effects become more complex and create a vicious circle.

Hepatocellular carcinoma, as previously related in this article, is an increasing concern in NAFLD. The annual incidence rate of developing HCC in patients with NASH-related cirrhosis range from $2.6 \%-12.8 \%$, but a significant proportion of patients with non-alcoholic fatty liver disease and NASH progress to HCC in the absence of cirrhosis ${ }^{(31,32)}$, what was observed in around one third of the cases in the Brazilian cohort ${ }^{(32)}$.

Even after the exclusion of HCC, malignancy is an important cause of death among NASH patients ${ }^{(21,22)}$. In a community-based cohort study in T2DM patients with and without NAFLD, the number of deaths due to malignancy were nearly the double in the former group when compared with those diabetics without NAFLD ${ }^{(19)}$. The colorectal region, esophagus, stomach, and pancreas are the main gastrointestinal sites of primary malignancy more frequently found while kidney (in men) and breast (in women) are the main extra gastrointestinal sites ${ }^{(19,21,22,33,34)}$. As most of these malignancies, as NAFLD, are related to obesity, diabetes and metabolic syndrome, it is not clear whether NAFLD is an additional risk for the development of such tumors, but recent evidence have raised concerns that NAFLD may be a new, and added, risk factor for extra-hepatic cancers, particularly in the gastrointestinal tract, most notably for colorectal cancer ${ }^{(34)}$. In fact, patients with NAFLD undergoing screening colonoscopy reveal significantly more colorectal cancer precursor lesions (adenomas) and early carcinoma compared with subjects without NAFLD ${ }^{(35)}$.

In the last years evidences linking NAFLD to the development and progression of chronic kidney disease (CKD) are emerging in increasing number in the literature ${ }^{(36-38)}$. NAFLD through exacerbate insulin resistance and release of proinflammatory and prothrombotic molecules can promote vascular and renal damage ${ }^{(37)}$. In the metaanalysis performed by Musso et al. ${ }^{(38)}$, NAFLD increased 2.12 times the risk of prevalent and 1.79 times the risk of incident CKD, and both incidence and prevalence increased progressively from simple steatosis to NASH and from NASH to advanced fibrosis, even after the adjustment for diabetes and others confounding factors.

So, accumulating evidence indicate that the effects of NAFLD extend beyond the liver and are associated with a range of chronic diseases, especially CVD, diabetes mellitus type 2 (T2DM) and CKD. It is becoming clear that although these diseases are the result of the same underlying pathophysiological processes associated with metabolic syndrome, they have been shown to be independent reciprocal risk factors and can act together to increase the risk of development of malignancies. In addition, recent data have shown that NAFLD actively contributes to aggravation of the pathophysiology of CVD, T2DM, and CKD, as well as several other diseases. NAFLD much more than a liver disease should be considered a multisystem disease, and broad internal medicine knowledge will be required for those doctors who intent to take care of these patients.

Edison Roberto PARISE*

Parise ER. Doença hepática gordurosa não alcoólica, mais que uma doença do fígado. Arq Gastroenterol. 2019;56(3):243-5.

DESCRITORES - Hepatopatia gordurosa não alcoólica. Carcinoma hepatocelular. Diabetes mellitus tipo 2. Doenças cardiovasculares. Insuficiência renal crônica.

\footnotetext{
* Universidade Federal de São Paulo (UNIFESP), Professor Associado da Disciplina de Gastroenterologia, São Paulo, SP, Brasil. Orcid: 0000-0003-4890-9259.
} 


\section{REFERENCES}

1. Younossi ZM, Stepanova M, Afendy M, Fang Y, Younossi Y, Mir H, Srishord M. Changes in the prevalence of the most common causes of chronic liver diseases in the United States from 1988 to 2008. Clin Gastroenterol Hepatol. 2011;9:524-30.

2. Younossi Z, Anstee QM, Marietti M, Hardy T, Henry L, Eslam M, et al. Globa burden of NAFLD and NASH: trends, predictions, risk factors and prevention. Nat Rev Gastroenterol Hepatol. 2018;15:11-20.

3. Williams CD, Stengel J, Asike MI, Torres DM, Shaw J, Contreras M, et al Prevalence of nonalcoholic fatty liver disease and nonalcoholic steatohepatitis among a largely middle-aged population utilizing ultrasound and liver biopsy: A prospective study. Gastroenterology. 2011;140:124-31.

4. Lazo M, Clark JM. The epidemiology of nonalcoholic fatty liver disease: a global perspective. Semin Liv Dis. 2008;28:339-50.

5. Leite NC, Villela-Nogueira CA, Cardoso CR, Salles GF. Non-alcoholic fatty liver disease and diabetes: from physiopathological interplay to diagnosis and treatment. World J Gastroenterol. 2014;20:8377-92.

6. Charlton MR, Burns JM, Pedersen RA, Watt KD, Heimbach JK, Dierkhising RA Frequency and outcomes of liver transplantation for nonalcoholic steatohepatitis in the United States. Gastroenterology. 2011;141:1249-53.

7. Banini BA, Mota M, Behnke M, Sharma A, Sanyal AJ. Nonalcoholic Steatohepatitis (NASH) has surpassed hepatitis $\mathrm{C}$ as the leading etiology for listing for liver transplant: Implications for NASH in Children and Young Adults. ACG Annual Scientific Meeting 2016, Abstract 46.

8. Wong RJ, Cheung R, Ahmed A. Nonalcoholic steatohepatitis is the most rapidly growing indication for liver transplantation in patients with hepatocellular carcinoma in the U.S. Hepatology. 2014;59:2188-95.

9. Cholankeril G, Yoo ER, Hu M, Gadiparthi C1, Khan MA1, Perumpail RB, et al. Rates of liver retransplantation in the United States are declining in the era of direct-acting antiviral agents. J Viral Hepat. 2017;24:1194-5.

10. Piñero F, Costa P, Boteon YL, Duque SH, Marciano S, Anders M, et al. A changing etiologic scenario in liver transplantation for hepatocellular carcinoma in a multicenter cohort study from Latin America. Clin Res Hepatol Gastroenterol. 2018;42:443-52.

11. Haldar D, Kern B, Hodson J, Armstrong MJ, Adam R, Berlakovich G, et al. Outcomes of liver transplantation for non-alcoholic steatohepatitis: A European Liver Transplant Registry study. J Hepatol. 2019;71:313-22.

12. Bugianesi E, Moscatiello S, Ciaravella MF, Marchesini G. Insulin resistance in nonalcoholic fatty liver disease. Curr Pharm Des. 2010;16:1941-51.

13. Cotrim HP, Parise ER, Oliveira CP, Leite N, Martinelli A, Galizzi J, et al. Nonalcoholic fatty liver disease in Brazil. Clinical and histological profile. Ann Hepatol. 2011;10:33-7.

14. Lazo M, Hernaez R, Eberhardt MS, Bonekamp S, Kamel I, Guallar E, et al Prevalence of nonalcoholic fatty liver disease in the United States: the Third National Health and Nutrition Examination Survey, 1988-1994. Am J Epidemiol. 2013;178:38-45.

15. Leite NC, Villela-Nogueira CA, Pannain VL, Bottino AC, Rezende GF, Cardoso $\mathrm{CR}$, Salles GF. Histopathological stages of nonalcoholic fatty liver disease in type 2 diabetes: prevalences and correlated factors. Liver Int. 2011;31:700-6.

16. Ekstedt M, Franzen LE, Mathiesen UL, Orelius L, Holmqvist M, Bodemar G, Kechagias S. Long-term follow-up of patients with NAFLD and elevated liver enzymes. Hepatology. 2006;44:865-73.

17. Sung KC, Wild SH, Byrne CD. Resolution of Fatty Liver and Risk of Incident Diabetes. J Clin Endocrinol Metab. 2013;98:363743.

18. Mantovani A, Byrne CD, Bonora E, Targher G. Nonalcoholic Fatty Liver Disease and Risk of Incident Type 2 Diabetes: A Meta-analysis Diabetes Care. 2018;41:372-82.

19. Adams LA, Harmsen S, St Sauver JL, Charatcharoenwitthaya P, Enders FB, Therneau T, Angulo P. Nonalcoholic fatty liver disease increases risk of death among patients with diabetes: a community based cohort study. Am J Gastroenterol. 2010;105:1567-73.
20. Ray G, Agarwala T. A study of metabolic parameters in nondiabetic patients with nonalcoholic fatty liver disease - importance of dyslipidemia. Arq Gastroenterol. 2019;56:270-5.

21. Adams LA, Lymp JF, St Sauver J, Sanderson SO, Lindor KD, Feldstein A, Angulo P. The natural history of nonalcoholic fatty liver disease: a population-based cohort study. Gastroenterology. 2005;129:113-21.

22. Angulo P, Kleiner DE, Dam-Larsen S, Adams LA, Bjornsson ES, Charatcharoenwithaya P, et al. Liver Fibrosis, but No Other Histologic Features, Is Associated With Long-term Outcomes of Patients With Nonalcoholic Fatty Liver Disease. Gastroenterology. 2015;149:389-97.

23. Hagström H, Nasr P, Ekstedt M, Hammar U, Stål P, Hultcrantz R, Kechagias S. Fibrosis stage but not NASH predicts mortality and time to development of severe liver disease in biopsy-proven NAFLD. J Hepatol. 2017;67:1265-73.

24. Dulai PS, Singh S, Patel J, Soni M, Prokop LJ, Younossi Z, et al. Increased risk of mortality by fibrosis stage in nonalcoholic fatty liver disease: Systematic review and meta-analysis. Hepatology. 2017;65:1557-65.

25. Castro PCS, Alberton HCP, Pedroso MLA, Morsoletto DBG, Pissaia Junior A, Ivantes CAP. Evaluation of progression of hepatic fibrosis in a group of patients with non-alcoholic fatty liver disease accompanied for 10 years. Arq Gastroenterol. 2019;56:256-60.

26. Madan SA, John F, Pyrsopoulos N, Pitchumoni C. Nonalcoholic fatty liver disease and carotid artery atherosclerosis in children and adults. A meta-analysis. Eur J Gastroenterol Hepatol. 2015;27:1237-48.

27. Wu S, Wu F, Ding Y, Hou J, Bi J, Zhang Z. Association of non-alcoholic fatty liver disease with major adverse cardiovascular events: A systematic review and meta-analysis. Scientific Reports 2016 volume 6, Article number: 33386.

28. Targher G, Byrne CD, Lonardo A, Zoppini G, Barbui C. Nonalcoholic Fatty Liver Disease and Risk of Incident Cardiovascular Disease: A Meta-Analysis of Observational Studies. J Hepatol. 2016;65:589-600.

29. Ballestri S, Lonardo A, Bonapace S, Byrne CD, Loria P, Targher G. Risk of cardiovascular, cardiac and arrhythmic complications in patients with non-alcoholic fatty liver disease. World J Gastroenterol. 2014;20:1724-45.

30. Francque SM, van der Graaff D, Kwanten WJ. Non-alcoholic fatty liver disease and cardiovascular risk: Pathophysiological mechanisms and implications. J Hepatol. 2016;65:425-43.

31. Cholankeril G, Patel R, Khurana S, Satapathy SK. Hepatocellular carcinoma in non-alcoholic steatohepatitis: Current knowledge and implications for management. World J Hepatol. 2017;9:533-43

32. Cotrim HP, Oliveira CP, Coelho HSM, Alvares-da-Silva MR, Nabuco L, Parise ER, et al. Nonalcoholic steatohepatitis and hepatocellular carcinoma: Brazilian survey. Clinics (Sao Paulo) 2016;71:281-4.

33. Sørensen HT, Mellemkjaer L, Jepsen P, Thulstrup AM, Baron J, Olsen JH, Vilstrup H. Risk of cancer in patients hospitalized with fatty liver: a Danish cohort study. J Clin Gastroenterol. 2003;36:356-9.

34. Sanna C, Rosso C, Marietti M, Bugianesi E. Non-Alcoholic Fatty Liver Disease and Extra-Hepatic Cancers. Int J Mol Sci. 2016;17:717.

35. Stadlmayr A, Aigner E, Steger B, Lederer, Mayr A Strasser M, et al. Nonalcoholic fatty liver disease: an independent risk factor for colorectal neoplasia. J Clin Med. 2011;270:41-9.

36. Marcuccilli M, Chonchol M. NAFLD and Chronic Kidney Disease. Int J Mol Sci. 2016;17:562

37. Targher G, Byrne CD. Non-alcoholic fatty liver disease: an emerging driving force in chronic kidney disease. Nat Rev Nephrol. 2017;13:297-310.

38. Musso G, Gambino R, Tabibian JH, EkstedtM, Kechagias S, Hamaguchi M, et al. Association of Non-alcoholic Fatty Liver Disease with Chronic Kidney Disease: A Systematic Review and Meta-analysis. PLoS Med. 2014;11: e1001680. 